\title{
Mean Field Approximation is Exact in the Many- Component Limit of Potts Lattice Gauge Model
}

\author{
R. Kotecký \\ Department of Mathematical Physics, Charles University, Povltavská 1, Praha 8, Pelc-Tyrolka, \\ Czechoslovakia
}

Abstract. The statement in the title concerning free energy is proved for a version of mean field approximation with previously fixed temporal gauge.

\section{Introduction}

Mean field theory yields a useful qualitative insight into a phase structure of lattice gauge theories and it is believed to be reliable for sufficiently high dimensions of spacetime [1]. Indeed, some of its predictions were confirmed by Monte Carlo experiments [2]. On the other side the use of mean field theory for lattice gauge models remains to be rather suspicious: the gauge invariance is broken in its very formulation - an order parameter is introduced that is known to vanish in a lattice gauge theory [3].

Though believed to be accurate in the limit of infinite dimensions, the exactness of a mean field approximation in this limit has, to the author's knowledge, never been proved ${ }^{\star}$. Here we address a similar problem, but we investigate a many component limit instead. Namely, we consider a $q$-state Potts lattice gauge model $[4,5]$ in the limit $q \rightarrow \infty$ and show that the mean field calculation of its free energy becomes exact. A similar result was proved recently for the conventional Potts model by Pearce and Griffith [6]. The remarkable feature of the gauge generalization of the Potts model is that a mean field approximation is exact in the limit $q \rightarrow \infty$ only when formulated in a variant that amounts to fixing timelike variables and applying mean field only to remaining links. To the contrary when mean field is applied directly to all links, without previous gauge fixing, one gets a strictly larger free energy and a mean field approximation fails to be exact. Thus, contrary to the case of the infinite dimensions limit [1], the differences between these two formulations of a mean field theory are not washed out in the limit $q \rightarrow \infty$. The situation is in accordance with a feeling that a (gauge noninvariant) mean field approximation should match only a model with a previously broken gauge symmetry.

A gauge Potts model as well as corresponding mean field theories will be introduced in detail in the next section. Here we only state our main result:

\footnotetext{
* See Note added in proof
} 
Theorem. Let $f(\beta, q)$ be a free energy at an inverse temperature $\beta$ of $q$-state Potts lattice gauge model on $d$-dimensional spacetime lattice, $d \geqq 2$. Then

$$
f^{\infty}(\beta) \equiv \lim _{q \rightarrow \infty} f(\log q \cdot \beta, q)=\lim _{q \rightarrow \infty} f_{M F}(\log q \cdot \beta, q)=\min \left(-\frac{d(d-1)}{2}-\frac{1}{\beta},-\frac{d}{\beta}\right) .
$$

Here $f_{M F}$ is a free energy of the fixed temporal gauge formulation of mean field theory.

Note that the limiting free energy $f^{\infty}$ has a corner in its dependence on inverse temperature $\beta$. This suggests a first-order phase transition at $\beta_{c} \sim \frac{2}{d} \log q^{1}$. Let us stress, however, that the above theorem does not prove that a first-order transition really occurs for any finite $q$. In fact one may easily imagine a sequence of smooth functions tending to $f^{\infty}$. After all, the theorem holds also in two dimensions where the phase transition certainly does not occur. The actual existence of a first-order transition for finite large $q$ and $d \geqq 3$ was proved recently using more involved techniques [7].

The Theorem will be proved by establishing upper and lower bounds on the free energy of the model that coincide in the limit $q \rightarrow \infty$ and yield thus the exact leading behaviour. An easy part is the upper bound by mean field theory. We obtain this in Sect. 2. Section 3 is devoted to the derivation of a more difficult and rather technical lower bound. This bound will be proved for each $q \geqq 2$ and may thus be of some interest in itself. That's why we state it here as a separate statement:

Lemma 1. $f(\beta, q) \geqq-\frac{1}{\beta}-\frac{1}{\beta} \frac{d(d-1)}{2} \log \left(e^{\beta}+q^{2 / \alpha}\right)$.

\section{Mean Field Upper Bound}

The Potts lattice gauge model was introduced by Kogut [4] who investigated the $\frac{1}{q}$ expansion in its continuous time (Hamiltonian) version. Here we treat its Lagrangian form, the large $q$ expansion of which was studied recently by Ginsparg et al. [5].

We consider a $d$-dimensional (spacetime) hypercubic lattice. Following the convenient notation of [8] we label unit coordinate vectors by $(\hat{\mu}, \mu=0,1, \ldots d-1$. We pick $\hat{0}$ as a vertical (temporal) direction. Whenever $i \in \mathbb{Z}^{d}$ is a lattice site we denote a (nonoriented) link connecting sites $i$ and $i+\hat{\mu}$ by a pair $(i, \mu)$ and a plaquette bordered by links $(i, \mu),(i+\hat{\mu}, \hat{v}),(i+\hat{v}, \mu)$ and $(i, v)$ by a triple $(i, \mu, v), \mu<v$. Occasionally (when the exact position is not important) we shall denote links (respectively plaquettes) by $\ell$ (respectively $P$ ). To introduce the model one attaches to each link $\ell$ a spin $\sigma_{\ell}$ taking values in $\mathbb{Z}_{q}=\{0,1, \ldots q-1\}$. Given a configuration $\sigma=\left\{\sigma_{\ell}\right\}$ one introduces a plaquette variable $\sigma_{P}$ in the usual way: if $P \equiv(i, \mu, v)$ then $\sigma_{P}=\sigma_{(i, \mu)}+\sigma_{(i+\hat{\mu}, v)}-\sigma_{(i+\hat{v}, \mu)}-\sigma_{(i, v)}(\bmod q)$. Consider a hypercube $\Lambda$ consisting of $|\Lambda|$ lattice sites. A free energy $f_{\Lambda}(\beta, q)$ is introduced by

1 Such asymptotic behaviour of $\beta_{c}$ is in agreement with the value $\beta_{c}=\log (1+\sqrt{q})$ that is in the case $d=4$ suggested by selfduality [5] 


$$
\exp \left(-\beta|\Lambda| f_{\Lambda}(\beta, q)\right)=Z_{\Lambda}(\beta, q)=\sum_{\sigma_{\Lambda}} \exp \left(-\beta H_{\Lambda}\left(\sigma_{\Lambda}\right)\right)
$$

The sum is over all configurations in $\Lambda$ and the energy (i.e. euclidean lattice action) of the Potts gauge model is ${ }^{2}$

$$
H_{\Lambda}\left(\sigma_{\Lambda}\right)=-\sum_{P \cap \Lambda \neq \varnothing} \delta_{\sigma_{P}, 0}
$$

To compute variables $\sigma_{P}$ for plaquettes that reach out of $\Lambda$ one extends a configuration $\sigma_{A}$ by an arbitrarily a priori chosen boundary configuration on the outside of $\Lambda$. It is known that a free energy $f(\beta, q)$ in the thermodynamic limit of expanding volume $\Lambda$ exists and does not depend on the choice of boundary condition [10].

A mean field approximation yields an upper bound on the free energy $f$. In fact an easy consequence of the Jensen inequality is the Gibbs' variational inequality asserting that for each probability measure $\omega_{\Lambda}$ on the space of all configurations in $\Lambda$

$$
f_{\Lambda}(\beta, q) \leqq \omega_{\Lambda}\left(\frac{H_{\Lambda}}{|\Lambda|}\right)-s_{\Lambda}\left(\omega_{\Lambda}\right)
$$

Here $\omega_{\Lambda}\left(\frac{H_{\Lambda}}{|\Lambda|}\right)$ is a specific energy in state $\omega_{\Lambda}$ and $s_{\Lambda}\left(\omega_{\Lambda}\right)=-\frac{1}{|\Lambda| \beta} \sum_{\sigma_{\Lambda}} \omega_{\Lambda}\left(\sigma_{\Lambda}\right)$ $\log \omega_{\Lambda}\left(\sigma_{\Lambda}\right)$ is its specific entropy. In particular choosing a probability $\omega_{\Lambda}\left(\sigma_{\Lambda}\right)=$ $\prod \omega_{\ell}\left(\sigma_{\ell}\right)$ that treats each spin $\sigma_{\ell}$ independently, one gets a mean field approxima-

$\ell \subset \Lambda$
tion. Next we choose the measures $\omega_{\ell}\left(\sigma_{\ell}\right)$ that prefer $\sigma_{\ell}=0$ with probability $p \in\langle 0,1\rangle$ and distribute remaining values of $\sigma_{\ell}$ uniformly with probability $\frac{1-p}{q-1}$. The probability that a plaquette in $\Lambda$ is nonfrustrated $\left(\sigma_{P}=0\right)$ under this measure equals $\left(p^{2}+\frac{(1-p)^{2}}{q-1}\right)^{2}+\frac{1}{q-1}\left(1-p^{2}-\frac{(1-p)^{2}}{q-1}\right)^{2}$. This follows when observing that the event $\sigma_{(i, \mu, v)}=0$ is the same as the event $\sigma_{(i, \mu)}+\sigma_{(i+\mu, v)}=$ $\sigma_{(i+\hat{v}, \mu)}+\sigma_{(i, v)}(\bmod q)$ and the variable $\sigma_{(i, \mu)}+\sigma_{(i+\hat{\mu}, v)}(\bmod q)\left(\right.$ as well as $\sigma_{(i+\hat{v}, \mu)}+$ $\left.\sigma_{(i, v)}(\bmod q)\right)$ equals 0 with probability $p^{2}+\frac{(1-p)^{2}}{q-1}$ with the remaining $q-1$ values distributed uniformly. Using this we evaluate the right hand side of (1) taking into account that to each lattice site $i$ there corresponds $\frac{d(d-1)}{2}$ plaquettes $(i, \mu, v), \mu<v$ and $d$ links $(i, \mu)$. After minimizing with respect to $p$ we obtain in the thermodynamic limit

$$
\begin{aligned}
& f(\beta, q) \leqq f_{M F}(\beta, q)=\inf _{p \in\langle 0,1\rangle}\left\{\frac { d ( d - 1 ) } { 2 } \left[-\left(p^{2}+\frac{(1-p)^{2}}{q-1}\right)^{2}\right.\right. \\
& \left.\left.-\frac{1}{q-1}\left(1-p^{2}-\frac{(1-p)^{2}}{q-1}\right)^{2}\right]+\frac{d}{\beta}\left[p \log p+(1-p) \log \frac{1-p}{q-1}\right]\right\} .
\end{aligned}
$$

2 Note that one gets the Potts gauge model directly from a $\mathbb{Z}_{q}-\operatorname{model}[9]$ when replacing $\cos \left(2 \pi \frac{\sigma_{P}}{q}\right)$ by $\delta_{\sigma_{P}, 0}$, i.e. introducing the energy that favours more decidedly configurations with nonfrustrated plaquettes $\left(\sigma_{p}=0\right)$ and suppresses a spinwave phase of the $\mathbb{Z}_{q}$-models 
For large $q$ the mean field entropy increases as $\log q$ and to get a nontrivial large $q$ behaviour one has to rescale the inverse temperature $\beta$ by $\log q$ [6]. Eventually we get

$$
\begin{aligned}
f^{\infty}(\beta) & \equiv \lim _{q \rightarrow \infty} f(\log q \cdot \beta, q) \leqq \lim _{q \rightarrow \infty} f_{M F}(\log q \cdot \beta, q) \\
& =\inf _{p \in\langle 0,1\rangle}\left\{-\frac{d(d-1)}{2} p^{4}-\frac{d}{\beta}(1-p)\right\}=\min \left(-\frac{d(d-1)}{2},-\frac{d}{\beta}\right) .
\end{aligned}
$$

The last equality is a consequence of concavity of the function $-\frac{d(d-1)}{2} p^{4}-\frac{d}{\beta}(1-p)$

It turns out that the right hand side of (2) is not the best estimate of $f^{\infty}(\beta)$. In fact the value on the right hand side will decrease if we introduce another version of a mean field approximation. To do that we use the gauge invariance of the model to change each configuration $\sigma$ into a configuration $\tilde{\sigma}$ of the same energy but with zero link variable on all vertical links. Namely we rewrite the partition function as $Z_{\Lambda}(\beta, q)=q^{|\Lambda|} e^{0(|\partial \Lambda|)} \sum_{\tilde{\sigma}_{A}} e^{-\beta H_{A}\left(\tilde{\sigma}_{\Lambda}\right)}=q^{|\Lambda|} e^{0(|\partial \Lambda|)} \tilde{Z}_{\Lambda}(\beta, q)$, where the sum is restricted over all configurations that are zero on all vertical links (we shall mark such configurations by a tilde $-\tilde{\sigma}_{\Lambda}$ ). The number of elements in the gauge group comes out as $q^{|\Lambda|}$ and an error $O(|\partial \Lambda|)$ proportional to the area of boundary $\partial \Lambda$ emerges as a consequence of the restoring of an original boundary condition that may have been spoiled by a gauge transformation. By denoting the sum as $\tilde{Z}_{\Lambda}(\beta, q)$ we indicate that one may introduce a new model that differs from the previous one only by having a restricted space of configurations -i.e. by having the gauge fixed. Denoting $\tilde{f}(\beta, q)$ a free energy of this new model we get

$$
f(\beta, q)=-\frac{1}{\beta} \log q+\tilde{f}(\beta, q)
$$

Now $\tilde{f}(\beta, q)$ may be estimated by a mean field approximation similar to the way it was done before for $f(\beta, q)$. The only difference is that this time our trial measure $\tilde{\omega}_{\Lambda}$ lives on the restricted configuration space and we choose $\tilde{\omega}_{\Lambda}\left(\tilde{\sigma}_{\Lambda}\right)=$ $\prod_{\ell \text { horizontal }} \omega_{\ell}\left(\tilde{\sigma}_{\ell}\right)$. This eventually leads to an inequality

$$
\begin{aligned}
\tilde{f}^{\infty \subset A}(\beta) & =\lim _{q \rightarrow \infty} \tilde{f}(\log q \cdot \beta, q) \leqq \\
& \inf _{p \in\langle 0,1\rangle}\left\{-\frac{(d-1)(d-2)}{2} p^{4}-(d-1) p^{2}-\frac{d-1}{\beta}(1-p)\right\} \\
& \min \left(-\frac{d(d-1)}{2},-\frac{d-1}{\beta}\right) .
\end{aligned}
$$

Taking into account the equality (3), we get for $f^{\infty}(\beta)$ a bound

$$
f^{\infty}(\beta)=-\frac{1}{\beta}+\tilde{f}^{\infty}(\beta) \leqq \min \left(-\frac{d(d-1)}{2}-\frac{1}{\beta},-\frac{d}{\beta}\right),
$$

which is for small temperatures clearly stronger than the previous one (2). 
Actually it turns out that this bound is exact. This will be proved in the next Section.

\section{A Lower Bound}

A needed lower bound follows immediately from the following Lemma (which is equivalent to that stated in the Introduction).

Lemma 1. $\tilde{f}(\beta, q) \geqq-\frac{1}{\beta} \frac{d(d-1)}{2} \log \left(e^{\beta}+q^{2 / \alpha}\right)$.

Proof. Let us evaluate the partition function $\tilde{Z}_{A}$ collecting the terms that have the same sets $\mathscr{P}$ of nonfrustrated plaquettes and summing over all subsets $\mathscr{P}$ of the set of all plaquettes that intersect $\Lambda$. Denoting by $|\{\}|$ the number of elements of a set described in brackets and $\mathscr{L}$ the set of all horizontal links in $\Lambda$ we have

$$
\begin{aligned}
\tilde{Z}_{\Lambda} & =\sum_{\mathscr{P}} e^{\beta|\mathscr{P}|}\left|\left\{\tilde{\sigma}_{\Lambda} \mid P \in \mathscr{P} \Leftrightarrow \delta_{\tilde{\sigma}_{P}, 0}=1\right\}\right| \leqq \sum_{\mathscr{P}} e^{\beta|\mathscr{P}|}\left|\left\{\tilde{\sigma}_{\Lambda} \mid P \in \mathscr{P} \Rightarrow \delta_{\tilde{\sigma}_{P}, 0}=1\right\}\right| \\
& \leqq \sum_{\mathscr{P}} e^{\beta|\mathscr{P}|} q^{|\mathscr{L} \backslash \overline{\mathscr{L}}(\mathscr{P})|}
\end{aligned}
$$

where $\overline{\mathscr{L}}(\mathscr{P})$ is any set of horizontal links such that

i) for each configuration on $\mathscr{L} \backslash \overline{\mathscr{L}}(\mathscr{P})$ there is at most one (restricted) configuration $\tilde{\sigma}_{A}$ on $\Lambda$ such that $\delta_{\tilde{\sigma}_{P}, 0}=1$ whenever $P \in \mathscr{P}$.

Moreover, for each $\mathscr{P}$ we shall find quite a large set $\overline{\mathscr{L}}(\mathscr{P})$ fulfilling i). Namely $\overline{\mathscr{L}}(\mathscr{P})$ such that

ii)

$$
|\overline{\mathscr{L}}(\mathscr{P})| \geqq \frac{|\mathscr{P}|}{\frac{d}{2}}-k|\partial \Lambda|
$$

with some constant $k$ independent of $\mathscr{P} .{ }^{3}$ Once we have such set $\overline{\mathscr{L}}(\mathscr{P})$ we estimate

$$
\begin{aligned}
\tilde{Z}_{\Lambda} & \leqq \sum_{\mathscr{P}} e^{\beta|\mathscr{P}|} q^{|\Lambda|(d-1)}-\frac{|\mathscr{P}|}{\frac{d}{2}}+k|\partial \Lambda| \\
& \leqq q^{k|\partial \Lambda|} \sum_{|\mathscr{P}|=0}^{|\Lambda| d(d-1) / 2+k^{\prime}|\partial \Lambda|}\left(\begin{array}{c}
|\Lambda|(d(d-1) / 2)+k^{\prime}|\partial \Lambda| \\
|\mathscr{P}|
\end{array}\right)\left(e^{\beta}\right)^{|\mathscr{P}|}\left(q^{2 / d)}\right)^{|\Lambda| d(d-1) / 2-|\mathscr{P}|+k^{\prime}|\partial \Lambda|} \\
& =q^{k|\partial \Lambda|}\left(e^{\beta}+q^{2 / d}\right)^{|\Lambda| d(d-1) / 2+k^{\prime}|\partial \Lambda|},
\end{aligned}
$$

with $|\Lambda|(d(d-1) / 2)+k^{\prime}|\partial \Lambda|$ the number of plaquettes intersecting $\Lambda$. The statement of the Lemma then follows immediately.

What remains is to find an algorithm for the construction of $\overline{\mathscr{L}}(\mathscr{P})$.

Let us imagine we have constructed it already and we want to verify the condition i). We are thus given a configuration outside of $\overline{\mathscr{L}}(\mathscr{P})$ that is to be extended onto $\overline{\mathscr{L}}(\mathscr{P})$. To do it we order the set of lattice sites in $\Lambda$ lexicographically:

3 The case of $\mathscr{P}$ the set of all plaquettes in $\Lambda$ shows that the inequality ii) is the best possible as far as leading terms in $|\Lambda|$ are concerned. Indeed, in this case $\overline{\mathscr{L}}(\mathscr{P})=\mathscr{L}$ since the configuration is determined everywhere solely from the knowledge of a boundary condition and up to terms proportional to $|\partial \Lambda|$ the numbers $|\overline{\mathscr{L}}(\mathscr{P})|$ and $|\mathscr{P}|$ equal $(d-1)|\Lambda|$ and $\frac{d(d-1)}{2}|\Lambda|$, respectively 
$i \leqq j$ whenever $j=i+\sum_{\mu=0}^{d-1} l_{\mu} \hat{\mu}$ and the first nonvanishing $l_{\mu}$ is negative. Then we shall determine the configuration inductively, extending it onto $\overline{\mathscr{L}}^{i}(\mathscr{P})=$ $\{(i, \mu) \in \overline{\mathscr{L}}(\mathscr{P})\}$ at one step. Namely, let the configuration be known already on $\bigcup \overline{\mathscr{L}}^{j}(\mathscr{P})$ and on the links outside $\Lambda$ (in the first step only the boundary condition is given). We want to prescribe its extension onto $\overline{\mathscr{L}}^{i}(\mathscr{P})$. We proceed by another induction link by link using the fact that a link variable on the link treated at one time can be computed from the condition $\tilde{\sigma}_{P}=0$ if this link belongs to a plaquette $P \in \mathscr{P}$ such that the configuration on all remaining three links of $P$ has already been determined. ${ }^{4}$ A feature of the construction of $\bar{L}^{i}(\mathscr{P})$ for each $i$ should be the existence of such a plaquette at each step. To ensure this one should not only choose which links of $\mathscr{L}^{i}=\{(i, \mu) \in \mathscr{L}\}$ to incorporate into $\overline{\mathscr{L}}^{i}(\mathscr{P})$ but also order the chosen links in an appropriate way. Both we shall do now.

First of all we may safely include into $\overline{\mathscr{L}}^{i}(\mathscr{P})$ the set $\overline{\mathscr{L}}_{1}^{i}(\mathscr{P})=\{(i, \mu) \mid(i, 0, \mu) \in \mathscr{P}\}$ ordered arbitrarily since when extending our configuration each plaquette $(i, 0, \mu)$ shall have already determined the three link variables $\tilde{\sigma}_{(i, 0)}(=0), \tilde{\sigma}_{(i+\hat{\mu}, 0)}(=0)$ and $\tilde{\sigma}_{(i+\hat{0}, \mu)}$. Next the set $\overline{\mathscr{L}}_{2}^{i}(\mathscr{P})$ of those links $(i, \mu) \notin \overline{\mathscr{L}}_{1}^{i}(\mathscr{P})$, for which there exists a link $(i, v) \in \overline{\mathscr{L}}_{1}^{i}(\mathscr{P})$ with corresponding plaquette $(i, \min (\mu, v), \max (\mu, v))$ belonging to $\mathscr{P}$, may also be included. (Again $(i, v),(i+\hat{\mu}, v)$ and $(i+\hat{v}, \mu)$ are the links on which the configuration will have been already determined.) To decide what additional links from $\mathscr{L}_{3}^{i}(\mathscr{P})=\mathscr{L}^{i}(\mathscr{P}) \backslash\left(\mathscr{L}_{1}^{i}(\mathscr{P}) \cup \mathscr{L}_{2}^{i}(\mathscr{P})\right)$ to include into $\overline{\mathscr{L}}^{i}(\mathscr{P})$ and how to order them, it is convenient to consider the following graph $G^{i}$. The set $\mathscr{L}_{3}^{i}(\mathscr{P})$ is taken to be the set of its vertices, and two vertices $(i, \mu),(i, v), \mu<v$ are joined by an edge whenever $(i, \mu, v) \in \mathscr{P}$. We shall use a simple graph-theoretical

Lemma 2. Let $G$ be a graph (without loops or multiple edges) having $r$ vertices and p edges. Then there exists a subset $\bar{V}$ of $k$ of its vertices and a linear ordering of $\bar{V}$ such that a) each vertex $v \in \bar{V}$ is joined by at least one edge with a vertex $w$ such that either $w \notin V$ or $w<v$,

b) $r \cdot k \geqq 2 p$.

Proof. Let $G$ have $f$ connected components. In each component we choose a path going through all vertices of the component. We omit the first vertex on the path, and the remaining vertices of the component we include into $V$, ordering them in the order we meet them for the first time on the path. The order of vertices from different components is given by an arbitrarily chosen ordering of components. The condition a) obviously holds. To verify b) observe that the number of edges of $G$ does not exceed the largest possible number of edges of a graph with $r$ vertices minus $f-1$ edges for each vertex that would join it with $f-1$ remaining components: $p \leqq \frac{r(r-1)}{2}-\frac{r(f-1)}{2}$. The condition b) then follows since $k=r-f$ by construction.

4 Let us note that any overdetermination does not bother us. We may fail to extend the configuration when two different plaquettes from $\mathscr{P}$ determine different values of a link variable on the same link, but this means only that the considered configuration cannot be extended. Actually, what we are looking for is the set $\overline{\mathscr{L}}(\mathscr{P})$ such that at most one extension exists 
To finish the proof of Lemma 1 we apply the above Lemma to the graph $G^{i}$ : we choose $\overline{\mathscr{L}}_{3}^{i}(\mathscr{P}) \subset \mathscr{L}_{3}^{i}(\mathscr{P})$ and order it in such a way that

a) each link $(i, \mu) \in \overline{\mathscr{L}}_{3}^{i}(\mathscr{P})$ is connected by a least one plaquette from $\mathscr{P}$ with a link $(i, v)$ that either does not belong to $\overline{\mathscr{L}}_{3}^{i}(\mathscr{P})$ or it is placed before $(i, \mu)$ in the order of $\overline{\mathscr{L}}_{3}^{i}(\mathscr{P})$.

Using this we easily see that we will be able to finish by induction the extension of our configuration onto

$$
\overline{\mathscr{L}}^{i}(\mathscr{P})=\overline{\mathscr{L}}_{1}^{i}(\mathscr{P}) \cup \overline{\mathscr{L}}_{2}^{i}(\mathscr{P}) \cup \overline{\mathscr{L}}_{3}^{i}(\mathscr{P}) .
$$

To verify ii) we show that for each lattice site $i$ that does not lie at the boundary of $\Lambda$ it holds $d\left|\overline{\mathscr{L}}^{i}(\mathscr{P})\right| \geqq 2\left|\mathscr{P}^{i}\right|$, where $\mathscr{P}^{i}=\{(i, \mu, v) \in \mathscr{P}\}$. Let us decompose disjointly $\mathscr{P}^{i}=\mathscr{P}_{1}^{i} \cup \mathscr{P}^{i}{ }_{2} \cup \mathscr{P}_{3}^{i}$ with $\mathscr{P}_{1}^{i}=\{(i, 0, \mu) \in \mathscr{P}\}, \mathscr{P}^{i}{ }_{3}=\{(i, \mu ; v) \in \mathscr{P} \mid(i, \mu) \notin$ $\left.\overline{\mathscr{L}}_{1}^{i}(\mathscr{P}),(i, v) \notin \overline{\mathscr{L}}_{1}^{i}(\mathscr{P})\right\}$ and $\mathscr{P}_{2}^{i}=\mathscr{P}^{i} \backslash\left(\mathscr{P}_{1}^{i} \cup \mathscr{P}^{i}\right)$. Clearly $\left|\overline{\mathscr{L}}_{1}^{i}(\mathscr{P})\right|=\left|\mathscr{P}_{1}^{i}\right|$. Since each plaquette $(i, \mu, v) \in \mathscr{P}_{2}^{i}$ has both links $(i, \mu)$ and $(i, v)$ in $\overline{\mathscr{L}}_{1}^{i}(\mathscr{P}) \cup \overline{\mathscr{L}}_{2}^{i}(\mathscr{P})$ while each of them belongs to at most $d-2$ (horizontal) plaquettes from $\mathscr{P}^{i}{ }_{2}$ we have $(d-2)$. $\left|\overline{\mathscr{L}}_{1}^{i}(\mathscr{P}) \cup \overline{\mathscr{L}}_{2}^{i}(\mathscr{P})\right| \geqq 2\left|\mathscr{P}_{2}^{i}\right|$, and as a consequence $d\left|\overline{\mathscr{L}}_{1}^{i}(\mathscr{P}) \cup \overline{\mathscr{L}}_{2}^{i}(\mathscr{P})\right| \geqq 2\left|\mathscr{P}_{1}^{i} \cup \mathscr{P}_{2}^{i}\right|$. To finish the proof we observe that from the condition b) of the Lemma 2 applied to the graph $G^{i}$ and from the fact that $\left|\mathscr{L}_{3}^{i}(\mathscr{P})\right|<d$ it follows that $d\left|\overline{\mathscr{L}}_{3}^{i}(\mathscr{P})\right| \geqq$ $\left|\mathscr{L}_{3}^{i}(\mathscr{P})\right|\left|\overline{\mathscr{L}}_{3}^{i}(\mathscr{P})\right| \geqq 2\left|\mathscr{P}_{3}^{i}\right|$.

Acknowledgements. It is pleasure to thank P. Holicky and D. Preiss for careful reading of the manuscript

Note added in proof: For the conventional Ising model, the exactness of the mean field theory in the limit of infinite dimensions was proven by Thompson [11]. (I thank Barry Simon for bringing this result to my attention.)

\section{References}

1. Balian, R., Drouffe, J. M., Itzykson, C. : Phys. Rev. D10, 3376-95 (1974), Drouffe, J. M., Itzykson, C. : Phys. Rep. 38C, 134-75 (1978)

2. Creutz, M., Jacobs, L., Rebbi, C. : Phys. Rev. Lett. 42, 1390-93 (1979), Phys. Rev. D20, 1915--22 (1979)

3. Elitzur, S. : Phys. Rev. D12, 3978-82 (1975)

4. Kogut, J. B. : Phys. Rev. D21, 2316-26 (1980)

5. Ginsparg, P., Goldschmidt, Y. Y., Zuber, J.-B. : Nucl. Phys. B170 FS1, 409-32 (1980)

6. Pearce, P. A., Griffiths, R. B.: J. Phys. A: Math. Gen. 13, 2143-48 (1980)

7. Kotecky, R., Shlosman, S. : First-order phase transitions in large entropy lattice models. (to be published)

8. Wilson, K. G. : Phys. Rev. D10, 2445-59 (1974)

9. Elitzur, S., Pearson, R. B., Shigemitsu, J. : Phys. Rev. D19, 3698-714 (1979)

10. Ruelle, D. : Statistical Mechanics (Rigorous Results). Chap. 2. New York-Amsterdam: Benjamin 1969. Israel, R. B. : Convexity in the Theory of Lattice Gases. Chap. 1. Princeton: Princeton University Press 1979

11. Thompson, C. J. : Commun. Math. Phys. 36, 255-262 (1974)

Communicated by K. Osterwalder

Received May 4, 1981 
